\section{F. Grémy}

Faculté de Médecine de MontpellierNîmes, France

\section{Preface}

\title{
Medical Informatics as a Set of Human Sciences? Why Not?
}

It is an honor to be invited to write the preface of the third IMIA Yearbook of Medical Informatics, in the footsteps of the recent presidents of IMIA, my good friends J.L. Willems and M.J. Ball. Compared with them, I feel like an MI "veteran", not to say an MI "has been", wondering whether I am a paleo- or just an archeo-medical informatician.

Let me take the privilege of looking at a science to which I have contributed, from both the point of view of history (over more than thirty years) and from an epistemological viewpoint (I am now mainly involved in public health).

A quick overview of the contents of the Yearbook clearly indicates the contrast between two developments: the tremendous progress of computer science and technology, and the painful complaint of many authors: why are our "chefs d'oevre" not used in routine practice? Why are most medical records still paper-based records, despite all their well-known drawbacks? And we do not understand why our wonderful decision-aiding tools have not succeeded in overcoming the resistance to adopt them in daily use. This is now the main concern of Artificial Intelligence in the medical com- munity which, according to Shortliffe, is "searching its soul".

The kind of solution suggested by many authors to overcome this situation is nearly always the following: let us make our tools more effective and, to achieve that, let us add improved technical tools (statistics, databases, information retrieval, etc.). But is the development of technology the most relevant and effective solution? One has reasonable doubts about this.

Looking back on the history of MI, I feel like quoting the famous British epidemiologist, A. Cochrane. Speaking of the health systems and their effectiveness, he compared them to the smoke coming out of the chimney of a crematorium: "so little out of so much". Dare we say the same about medical informatics - after more than thirty years of international efforts, after myriads of publications, seven MEDINFO congresses and 17 SCAMC meetings (the last one, in November 1993 , drew nearly 3,000 people to Washington)? What are the causes of such delusions? Probably in our poor reflection about the respective place of man and machine in our programs.

At the last SCAMC meeting, I had 
the privilege and honor to refute the claim of informatics to be a substitute for the human mind. Informatics in general, and more particularly medical informatics, is in great need of a philosophy, just to debunk a "misleading and arrogant conception of the socalled "intellectual" capabilities of computer machinery". As Houziaux wrote very wisely: "Informatics progresses on a field of lexical mines". I pleaded that, indeed, many of the keywords of informatics "memory, language, knowledge, intelligence, etc." are just epistemological catchwords, and that "any idea of comparison between human mind and electronic computer is misleading and should be used with extreme caution ...", and that "any idea of competition is definitely absurd".

Beside this deficit in philosophy and epistemology, informatics also experiences deficits in human sciences: psychology, linguistics, sociology.

If we reject the idea that a computer is able to duplicate human intelligence and, moreover, human compassion, we cannot ignore that informatics is strongly connected with the human mind, and that it is able to provide significant help to enlarge its capabilities.

An insufficient study of how these complementary entities work, which intellectual and emotional constraints are demanded from the human user - in an interaction which is not a dialogue (another catchword) - is probably the main cause of failures, especially in clinical practice. Informatics gives rise to both fantasies and disappointments. The psychology of man-machine interaction is probably not well known, especially when the user is no more than a layman.

On the other hand, too often medical informaticians - more technician than medical - offer a priori designed and ready-made solutions, without investigation of the needs and the ergonomy of medical practice.
How does the clinic work? or a group of clinicians during a ward visit? - the information they ask for and how they use it, etc. is a domain for psychological exploration (objective recording, questionnaires, interviews). A similar situation can be envisaged from the patient's viewpoint. The presence of good psychologists in both the medical team and the informatician team is probably the solution to avoid the double pitfall of unquestioned answers, and unanswered questions.

At another level, when the functioning of a large institution (such as a hospital) implies the management of large flows of information, one should keep in mind that a lack of social sciences, and especially in organizations, may explain the difficulties in establishing an efficient and well-accepted Hospital Information System. Winograd and Flores have shown that such institutions are not "information systems" in the technical meaning of the word, but "conversation systems"; in this case the role of a computer system is to facilitate those conversations, instead of imposing heavy constraints on the personnel. Peterson said with humor: "Do not computerize the hospital. Hospitalize the computer". How many computerized Hospital Information Systems run satisfactorily throughout the world, despite the mass of essays, papers and books published?

These considerations lead to two sets of interrogations:

1. The unity of Medical Informatics,

2. The place of human beings in the medical and health process has been greatly underestimated and must be restored. Also, one must look at Medical Informatics - at least some parts of it - as a human science or, more exactly, a set of human sciences.

Medical Informatics (or Health Informatics) is often claimed as a new medical (health) discipline. It is not clear that this is so.

Has it any sense to found a discipline on a tool and its applications to a given domain? If so, this means that the application domain (in this case medicine) is a source of progress for the tools (here computer scierices). It implies also that the use of the tool has potential for modifying the application domain. A third implication is that the combination of the tool and the domain of application gives rise to specific realizations, and needs specially trained personnel. A fourth implication is that, as soon as it is founded, the new discipline may not explode into several more or less separated subfields.

What about medical informatics? Let us consider another applied discipline, 20 years older than medical informatics: medical biostatistics, which seems to meet the above-mentioned criteria. Biostatistics has kept a specificity and a unity, independent of the disciplines it has given birth to: clinical research methodology and epidemiology. There is room for further theoretical research in medical biostatistics, which is itself a well-defined part of statistical science. So, it is not surprising to find in some American schools of public health, distinct departments devoted to epidemiology and biostatistics.

Does the same apply to medical and health informatics? It seems that medical applications have not been occasions for significant progress in computer science (except, perhaps, in Artificial Intelligence: medical AI applications have been more useful for AI than for medicine). Has medical informatics modified the practice of medicine? Too little: the routine use of computerized medical records, of computer-aided medical training, of decision-support systems, remains more of a hope than a reality.

The unity of medical informatics should also be discussed: one can di- 
vide most of medical informatics into four domains:

1. imaging,

2. aid to clinical action, reasoning, evaluation and education,

3. aid to management of health institutions (such as hospitals),

4. general health statistics.

The disparity of those domains becomes obvious when one looks at the evaluation criteria for the respective applications.

In the general background of any evaluation in computer sciences - does the program do what it is supposed to do? One finds that domains 1 and 4 have in common that they provide facts (images, health indices, etc.), impossible to obtain without enormous computing (in the narrow meaning of the word) capabilities. Based on these facts, health professionals can reason as with any other kind of facts. Computer technology is mandatory, but is inferior to the professional intellectual process, and remains external to it.

For the two other domains, informatics is supposed to closely intermingle with human intellectual activities. Evaluation in domain 2 demands reference to epistemology and linguistics on the one hand, and to different aspects of psychology on the other (cognitive, instruction, even emotional psychology).

As for domain 3, sociological and psycho-sociological concerns are basic constituents of Hospital information Systems. The success or failure of such systems depends more on their acceptability than on specific technical properties, and when one compares how users on the one hand and designers of the system on the other consider the information system, the evidence of huge discrepancies explains the difficulties of the system. A recent sociological study conducted by $\mathrm{M}$. Bonnin in my laboratory is very convincing in this respect.

Finally, domains 1 and 4 can be developed and evaluated without a strong involvement of human sciences: the computer is no more than a tool. But domains 2 and 3 deal mainly with human behavior and acceptance. Computer technology hides behind human reactions.

This feature threatens both the unity and the specificity of medical informatics. The aim of this paper is to stimulate reflection and discussion. Why not a working conference, or a special session in the next MEDINFO to discuss these fundamental issues of our Medical Informatics Science(s)?

Address of the author:

Prof. François Grémy,

Faculté de Médecine de Montpellier-Nîmes, Hôpital Lapeyronie, SEMHAP,

371 Av. du Doyen Giraud, 34295 Montpellier Cedex 5,

France 